\title{
On the Estimation of Longitudinal Dynamics of Powered Two-Wheeled Vehicles
}

\author{
Mohammed El-Habib Dabladji, Dalil Ichalal, Hichem Arioui and Saïd Mammar \\ Informatics, Integrative Biology and Complex Systems Laboratory (IBISC EA-4526) \\ Université d'Évry Val d'Essonne, 91020 Évry, France.
}

\begin{abstract}
A robust observer is presented in this paper to estimate the longitudinal tire-road forces, the engine and braking torques for powered two-wheeled vehicles. A study of detectability of the longitudinal dynamics is presented and the tire-road longitudinal forces are estimated using an unknown input observer without any knowledge of tire parameters. The effectiveness of the proposed approach is shown through simulation results.
\end{abstract}

\section{INTRODUCTION AND PROBLEM STATEMENT}

Development of safety systems for powered two-wheeled vehicles (P2WV) is an important issue due to the injuries and the significant number of fatalities worldwide. Compared to standard vehicles, several elements may explain this delay in the development of safety systems for P2WV: the low market of P2WV and the investments on R\&D which are limited. Moreover, users' acceptance and freedom feeling is also a key point that is often neglected. Finally, P2WV are very hard to control due to the complex dynamic behaviors which are naturally unstable and which are sensitive to riders' movements [1].

In this context, our long term objective concerns the design of passive and active safety systems by using the automatic control theory. However, this needs the knowledge of the vehicle's dynamics. Unfortunately, some variables are not available with conventional sensors for economic and technical reasons. To overcome these problems, constructing observers to estimate dynamic variables of $\mathrm{P} 2 \mathrm{WV}$ is essential in perspective of passive or active assistance systems design. In the field of observer design for such vehicles, there are only few techniques since the domain is new. Nevertheless, one can cite the design and implementation of an Extended Kalman Filter (EKF) [2] which takes into account the nonlinear behavior of the system and some stochastic measurement noises. More recently, High Order Sliding Mode technique has been used to estimate rider's torque and the roll angle by using a linearized model of the vehicle [3] with constant longitudinal velocity. Nonlinear approaches based on Takagi-Sugeno model are developed in [4], [5] which provide nonlinear observers taking into account several important nonlinear behaviors, especially, those related to the roll motion and the time varying longitudinal velocity. All these techniques are based on linear or nonlinear tire-road forces' models (e.g. Pacejka's model [6]) which are empirical models, thus, uncertain.

In this paper, an approach to estimate the longitudinal dynamics of $\mathrm{P} 2 \mathrm{WV}$ is proposed. It is based on a linear
Proportional-Derivative unknown input observer and the Lyapunov theory where we need any model of the tire forces. $H_{\infty}$ criterion is added to make the observer less sensitive to some inputs and measurements and also to make it more robust to uncertainties.

It is well known in motorcycle and bicycle community that tire forces are very hard to measure or to estimate ; and at the same time, they are seen to be the most important variables that affect the comfort and safety of riders [7]. In this context, it seems important to estimate the longitudinal forces in their nonlinear form even if the pneumatic parameters are unknown. Accordingly, to be more consistent with realistic constraints, we consider the longitudinal tire forces and the engine or braking torques as unknown inputs and we try to reconstruct them directly thanks to the proposed observer. The paper is organized as follows. Section [I briefly presents P2WV's longitudinal dynamics. Section III recalls the detectability of P2WV's longitudinal model. Section IV describes the proposed nominal and robust observer. Finally, section $\square$ provides some simulation results.

\section{TWO-WHEELED LONGITUDINAL DYNAMICS DESCRIPTION}

This section is devoted to the modeling of the longitudinal dynamics. After, we will propose two observers to estimate the longitudinal forces. The only restriction for the observation part is to know the ratio of braking torque on the two tires during the deceleration phase, and to suppose that during acceleration phase, the engine torque is applied only to rear wheel. An analytic model of the longitudinal dynamics is derived from the single-corner model [7], [8] and is given by the following equations (see also figure 1 ):

$$
\left\{\begin{aligned}
M a_{x} & =F_{x f}+F_{x r}-C_{d} v_{x}^{2} \\
i_{f y} \dot{\omega}_{f} & =-R_{f} F_{x f}-B_{f} \\
i_{r y} \dot{\omega}_{r} & =-R_{r} F_{x r}+E-B_{r}
\end{aligned}\right.
$$

$B_{f}$ and $B_{r}$ are the braking torques applied to the front and the rear wheel respectively. $E$ is the engine torque. We suppose that the engine torque is applied only to the rear wheel. $v_{x}$ and $a_{x}$ are the longitudinal velocity and acceleration. $F_{x f}$ and $F_{x r}$ refer to the front and rear longitudinal forces. $\omega_{f}$ and $\omega_{r}$ are the front and rear wheel angular speeds. The front and rear tire radii are $R_{f}$ and $R_{r}$ and $i_{f y}$ and $i_{r y}$ mean the front and rear rotational inertia. $M=M_{m}+M_{r}$ is the sum of the 


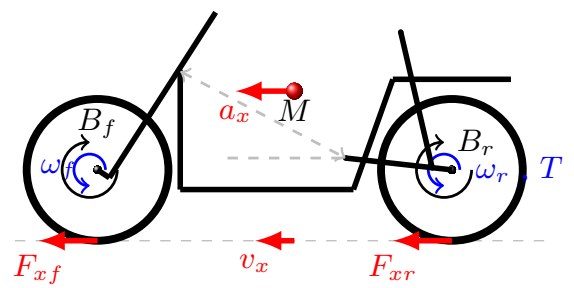

Fig. 1. Representation of a P2WV model with longitudinal dynamics

P2WV's mass and the rider's mass. Finally, $C_{d}$ refers to the drag aerodynamic coefficient.

The front and rear braking are assumed to be coupled and the ratio of braking torque between the two wheels is considered known. We consider $B_{f}=\alpha B$ and $B_{r}=(1-\alpha) B$ with $\alpha \in[0,1]$ and $B$ is the new braking torque to distribute between the two wheels. Thus, the model (1) can be writing in the following form:

$$
\left\{\begin{aligned}
M a_{x} & =F_{x f}+F_{x r}-C_{d} v_{x}^{2} \\
i_{f y} \dot{\omega}_{f} & =-R_{f} F_{x f}+\alpha T \\
i_{r y} \dot{\omega}_{r} & =-R_{r} F_{x r}+(1-\alpha) T
\end{aligned}\right.
$$

where: $T=\left\{\begin{array}{cc}E & \text { acceleration phase } \\ -B & \text { braking phase }\end{array}\right.$ and $\alpha=\left\{\begin{array}{cc}0 & \text { acceleration phase } \\ \alpha^{*} & \text { braking phase }\end{array}\right.$

and $\alpha^{*}$ is ratio of braking torque between the two wheels.

It is easy to measure the longitudinal acceleration thanks to accelerometer, and the wheels angular speed with optical encoders. In fact, these sensors are available on motorcycles equipped with anti-lock braking systems (ABS). Thus, the more difficult task consists on the estimation of the longitudinal forces. This is the aim of our work.

\section{Study of Detectability}

Before speaking about the estimation of the longitudinal dynamics, let us recall some definitions about the unknowninput (UI) observability and detectability of nonlinear systems. The UI observability (detectability) relates to the possibility of reconstruct the UI uniquely infinite-time (asymptotically) having as information the known inputs and outputs.

Definition 1: [9] Consider the following nonlinear system with $x(t)$ the state vector, $u(t)$ the known inputs vector, $w(t)$ the UI vector and $y(t)$ the measurements vector:

$$
\left\{\begin{array}{l}
\dot{x}(t)=f(x(t), u(t), w(t)) \\
y(t)=h(x(t), u(t), w(t))
\end{array}\right.
$$

For every initial condition $x(0)$ and any known input $u(t)$, the nonlinear system (3) is called:

- state and UI strongly observable, if: $y(t, x(t), u(t), w(t))=y(t, \bar{x}(t), u(t), \bar{w}(t))$ implies that: $x(t)=\bar{x}(t)$ and $w(t)=\bar{w}(t)$.

- state and UI strongly detectable, if: $y(t, x(t), u(t), w(t))=y(t, \bar{x}(t), u(t), \bar{w}(t))$ implies that: $x(t) \rightarrow \bar{x}(t)$ and $w(t) \rightarrow \bar{w}(t)$ as $t \rightarrow \infty$.
- state strongly asydetectable, if: $y(t, x(t), u(t), w(t)) \rightarrow y(t, \bar{x}(t), u(t), \bar{w}(t))$ as $t \rightarrow \infty$ implies that: $x(t) \rightarrow \bar{x}(t) w(t) \rightarrow \bar{w}(t)$ as $t \rightarrow \infty)$.

Let us check the UI strong observability (detectability or asydetectability) of the model giving by (1). We consider that the longitudinal dynamics state vector $\left(\left[\omega_{f}(t), \omega_{r}(t)\right]^{T}\right)$ is measurable. Moreover, since $a_{x}$ and $v_{x}$ are considered measurable, then we can say that $F_{x f}+F_{x r}$ is also known from (1). So, the vector $z(t)=\left[\omega_{f}(t), \omega_{r}(t), F_{x f}+F_{x r}\right]^{T}$ is known.

Now, from definition 1, consider the trajectory of the system (1) and another trajectory:

$$
\left\{\begin{aligned}
M \bar{a}_{x} & =\bar{F}_{x f}+\bar{F}_{x r}-C_{d} \bar{v}_{x}^{2} \\
i_{f y} \dot{\bar{\omega}}_{f} & =-R_{f} \bar{F}_{x f}-\bar{B}_{f} \\
i_{r y} \dot{\bar{\varphi}}^{2} & =-R_{r} \bar{F}_{x r}+\bar{T}-\bar{B}_{r}
\end{aligned}\right.
$$

and suppose that $z(t)=\bar{z}(t)$. Thus, we obtain:

$$
\left\{\begin{array}{r}
\tilde{F}_{x f}+\tilde{F}_{x r}=0 \\
-R_{f} \tilde{F}_{x f}-\tilde{B}_{f}=0 \\
-R_{r} \tilde{F}_{x r}+\tilde{T}-\tilde{B}_{r}=0
\end{array}\right.
$$

where: $\tilde{x}$ refers to $x-\bar{x}$.

We see that the obtained system has $\left(\tilde{F}_{x f}+\tilde{F}_{x r}\right)=0$ as unique solution, but $\left(\tilde{F}_{x f}, \tilde{F}_{x r}\right)=(0,0)$ is not the unique one. Then, the longitudinal forces cannot be observable with such a model. This motivates our constraints to consider the ratio of braking torque to be known.

In the case where this ratio is known, we obtain the following system of equation:

$$
\left\{\begin{array}{r}
\tilde{F}_{x f}+\tilde{F}_{x r}=0 \\
-R_{f} \tilde{F}_{x f}+\alpha \tilde{T}=0 \\
-R_{r} \tilde{F}_{x r}+(1-\alpha) \tilde{T}=0
\end{array}\right.
$$

Now, we see that the only solution of the above system of equations is $\left[\tilde{F}_{x f}, \tilde{F}_{x r}, \tilde{T}\right]^{T}=0$. So, if the ratio of braking torque is known, the longitudinal forces become strongly observable i.e. they will be estimated exactly.

\section{OBSERVER DESIGN}

To estimate the longitudinal forces, we consider that the longitudinal acceleration and velocity and the angular velocities of the wheels are measured. The first derivatives of the angular velocities of the wheels are also considered known. These derivatives can be obtained by the direct derivation of the angular velocities if the latter are not very noisy or already filtered. Otherwise, there are several techniques to estimate the derivative of signals such as the super-twisting algorithms [10].

From (2), the system can be writing in the following form:

$$
\begin{gathered}
\underbrace{\left(\begin{array}{ccc}
M & 0 & 0 \\
0 & i_{f y} & 0 \\
0 & 0 & i_{r y}
\end{array}\right)}_{M_{1}} \underbrace{\left(\begin{array}{c}
a_{x} \\
\dot{\omega}_{f} \\
\dot{\omega}_{r}
\end{array}\right)}_{D_{1}}= \\
\underbrace{\left(\begin{array}{ccc}
1 & 1 & 0 \\
-R_{f} & 0 & \alpha \\
0 & -R_{r} & 1-\alpha
\end{array}\right)}_{\dot{Y}} \underbrace{\left(\begin{array}{c}
F_{x f} \\
F_{x r} \\
T
\end{array}\right)}_{U}-\underbrace{\left(\begin{array}{c}
C_{d} \\
0 \\
0
\end{array}\right)}_{D_{2}} v_{x}^{2}
\end{gathered}
$$


$Y$ refers to $\left(v_{x}, \omega_{f}, \omega_{r}\right)^{T}$ and $\dot{Y}$ refers to its derivative $\left(a_{x}, \dot{\omega}_{f}, \dot{\omega}_{r}\right)^{T}$

Note that considering the tire forces as unknown inputs is more consistent with real constraints, because they depend on several parameters (tire pressure, tire adhesion, mass transfer between the two tires, etc.). In fact, it is not necessary in this paper to know the tire's parameters or dynamics.

Since the rotational tire velocities $\omega_{f}$ and $\omega_{r}$ and their first time derivatives are bounded, we use the super-twisting algorithms [10] to estimate the first derivatives $\dot{\omega}_{f}$ and $\dot{\omega}_{r}$

$$
\left\{\begin{array}{l}
\dot{v}_{11}=v_{12}-\lambda_{11}\left|v_{11}-\omega_{f}\right|^{\frac{1}{2}} \operatorname{sign}\left(v_{11}-\omega_{f}\right) \\
\dot{v}_{12}=-\lambda_{12} \operatorname{sign}\left(v_{12}-\dot{v}_{11}\right) \\
\dot{v}_{21}=v_{22}-\lambda_{21}\left|v_{21}-\omega_{r}\right|^{\frac{1}{2}} \operatorname{sign}\left(v_{21}-\omega_{r}\right) \\
\dot{v}_{22}=-\lambda_{22} \operatorname{sign}\left(v_{22}-\dot{v}_{21}\right)
\end{array}\right.
$$

sign refer to the sign function. $\lambda_{i j}$ are positive scalars and are chosen according to the limits of the derivatives of $\omega_{f}$ and $\omega_{r}$ (see [10]). $v=\left[v_{12}, v_{22}\right]^{T}$ are the estimates of $\left[\dot{\omega}_{f}, \dot{\omega}_{r}\right]^{T}$.

\section{A. Nominal observer for the longitudinal dynamics}

Under the assumption that the model of the longitudinal dynamics is well known, let us consider the following observer:

$$
\left\{\begin{array}{ccc}
M_{1} \dot{\hat{Y}} & =D_{1} \hat{U}-D_{2} v_{x}^{2}+K_{1} \tilde{Y} \\
\dot{\hat{U}} & = & K_{2} \tilde{Y}+K_{3} M_{1} \dot{\tilde{Y}}
\end{array}\right.
$$

where: $\tilde{Y}=Y-\hat{Y}$.

The gains of the observer are computed according to the following theorem:

Theorem 1: Under the assumption that the $1^{\text {st }}$ derivative of the unknown input vector is bounded and given positive diagonal matrices $Q_{1}$ and $Q_{2}$, if matrices $K_{1}, L_{2}$ and $K_{3}$ solution to the optimization problem (10) subject to the LMI (11) exist, then, $H_{\infty}$ weighting performance is guaranteed for the estimation of the state vector $Y$ and the unknown input $U$.

$$
\min _{K_{1}, L_{2}, K_{3}} \bar{\gamma}
$$

s.t.

$$
\left(\begin{array}{ccc}
-K_{1}-K_{1}^{T} & L_{2}^{T} & 0 \\
* & -K_{3} D_{1}-D_{1}^{T} K_{3}^{T}+Q_{1} & I \\
* & * & -\bar{\gamma} Q_{2}
\end{array}\right) \leq 0
$$

The gain $K_{2}$ is computed from: $K_{2}=D_{1}^{T}-L_{2}+K_{3} K_{1}$

The $H_{\infty}$ weighting performance is guaranteed w.r.t. $\dot{U}$, with the weighting matrices $Q_{1}$ and $Q_{2}$ and the attenuation level is $\gamma=\sqrt{\bar{\gamma}}$ which means that:

- if $\dot{U}=0$, then $: \tilde{Y} \rightarrow 0$ and $\tilde{U} \rightarrow 0$.

- if $\dot{U} \neq 0$, then : $\tilde{U}^{T} Q_{1} \tilde{U} \leq \gamma^{2} \dot{U}^{T} Q_{2} \dot{U}$.

Proof: From the P2WV's longitudinal model (7) and the observer structure (9), the dynamic of the estimation errors is given by:

$$
\begin{cases}M_{1} \dot{\tilde{Y}} & =D_{1} \tilde{U}-K_{1} \tilde{Y} \\ \dot{\tilde{U}} & =\dot{U}+\left(K_{3} K_{1}-K_{2}\right) \tilde{Y}-K_{3} D_{1} \tilde{U}\end{cases}
$$

We consider the following Lyapunov function:

$$
V=\tilde{Y}^{T} M_{1} \tilde{Y}+\tilde{U}^{T} \tilde{U}
$$

From the estimation errors dynamic (12), the time derivative of the Lyapunov function is as follows:

$$
\begin{aligned}
\dot{V} & =-\tilde{Y}^{T}\left(K_{1}+K_{1}^{T}\right) \tilde{Y}+2 \tilde{Y}^{T}\left(D_{1}-K_{2}^{T}+K_{1}^{T} K_{3}^{T}\right) \tilde{U} \\
& -\tilde{U}^{T}\left(K_{3} D_{1}+D_{1}^{T} K_{3}^{T}\right) \tilde{U}+2 \tilde{U}^{T} \dot{U}
\end{aligned}
$$

The main objective here is to attenuate the effect of the vector $\dot{U}$ on the error estimation of the unknown input $\tilde{U}$. The vector $\tilde{Y}$ is well known from the sensors, so it is not interesting to attenuate the effect of $\dot{U}$ on $\tilde{Y}$. Moreover, since the magnitudes of the elements of the vectors $\dot{U}$ and $\tilde{U}$ are not the same, The $H_{\infty}$ weighting performance with weighting matrices $Q_{1}$ and $Q_{2}$ w.r.t. $\dot{U}$ is guaranteed if and only if

$$
\dot{V}+\tilde{U}^{T} Q_{1} \tilde{U} \leq \gamma^{2} \dot{U}^{T} Q_{2} \dot{U}
$$

From the expression of the derivative of the Lyapunov function (14), the inequality (15) is equivalent to:

$$
\left(\begin{array}{ccc}
-K_{1}-K_{1}^{T} & D_{1}-K_{2}^{T}+K_{1}^{T} K_{3}^{T} & 0 \\
* & -K_{3} D_{1}-D_{1}^{T} K_{3}^{T}+Q_{1} & I \\
* & * & -\gamma^{2} Q_{2}
\end{array}\right) \leq 0
$$

By setting $\bar{\gamma}=\gamma^{2}$ and $L_{2}=D_{1}^{T}-K_{2}+K_{3} K_{1}$, the LMI below is the same as 111 given in the theorem below. So, by the minimization of $\bar{\gamma}$, we will minimize the effect of the vector $\dot{U}$ on the error estimation of the unknown input $\tilde{U}$.

Thus, with the proposed observer, we can estimate the longitudinal forces and the engine or braking torque without any knowledge of the tire parameters.

\section{B. Robust observer for the longitudinal dynamics}

The proposed observer given before was designed by considering the mass of the vehicle constant and known which is not the case because it can change with respect to the mass of the rider.

Moreover, the effective rolling radius of wheels $R_{f}$ and $R_{r}$ in free motion is smaller than the radius of the unloaded tire because of its deformation. The new values of the effective rolling radius depend on the forward velocity, tire radial stiffness, tire load and inflation pressure.

Also, the aerodynamics coefficient $C_{d}$ may be subject to uncertainties because it changes w.r.t. riding position.

So, the nominal observer proposed before doesn't take into account these uncertainties and we don't have any proof of convergence of the observer in the realistic case.

In this context, we propose in this part a robust observer to estimate the longitudinal dynamics of the P2WV whatever it is subject to uncertainties.

The P2WV longitudinal dynamics are modeled in this case as follows:

$$
\underbrace{\left(\begin{array}{ccc}
M_{0}+\delta M & 0 & 0 \\
0 & i_{f y} & 0 \\
0 & 0 & i_{r y}
\end{array}\right)}_{M_{10}+\delta M_{1}} \underbrace{\left(\begin{array}{c}
a_{x} \\
\dot{\omega}_{f} \\
\dot{\omega}_{r}
\end{array}\right)}_{\dot{Y}}=
$$




$$
\begin{gathered}
\underbrace{\left(\begin{array}{ccc}
1 & 1 & 0 \\
-R_{f 0}-\delta R_{f} & 0 & \alpha \\
0 & -R_{r 0}-\delta R_{r} & 1-\alpha
\end{array}\right)}_{D_{10}+\delta D_{1}} \underbrace{\left(\begin{array}{c}
C_{d 0}+\delta C_{d} \\
0 \\
0
\end{array}\right)}_{U} v_{D_{20}+\delta D_{2}}^{2}
\end{gathered}
$$

where: $|\delta M|<\Delta_{1},\left|\delta R_{f}\right|<\Delta_{2},\left|\delta R_{r}\right|<\Delta_{2}$ and $\left|\delta C_{d}\right|<$ $\Delta_{3}$.

$$
\begin{aligned}
& \delta M_{1}=\left(\begin{array}{ccc}
\delta M & 0 & 0 \\
0 & 0 & 0 \\
0 & 0 & 0
\end{array}\right), \delta C_{d}=\left(\begin{array}{c}
\delta C_{d} \\
0 \\
0
\end{array}\right), \\
& \delta D_{1}=\left(\begin{array}{ccc}
0 & 0 & 0 \\
-\delta R_{f} & 0 & 0 \\
0 & -\delta R_{r} &
\end{array}\right) .
\end{aligned}
$$

Since $\delta M, \delta R_{f}, \delta R_{r}$ and $\delta C_{d}$ are bounded, we can write: $\delta M_{1}=\Delta_{1} \delta_{1}, \delta D_{1}=\Delta_{2} \delta_{2}$ and $\delta C_{d}=\Delta_{3} \delta_{3}$; with: $\delta_{1}^{T} \delta_{1} \leq I, \delta_{2}^{T} \delta_{2} \leq I$ and $\delta_{3}^{T} \delta_{3} \leq I$.

The robust observer has the same structure as in the previous case (9). The gains of the observer in this case will be computed according to the following theorem:

Theorem 2: Under the assumption that the $1^{\text {st }}$ derivative of the unknown input vector and the uncertainties $\delta M, \delta R_{f}, \delta R_{r}$ and $\delta C_{d}$ are bounded. Given positive diagonal matrices $Q_{1}$, $Q_{21}, Q_{22}, Q_{23}, Q_{24}$ if matrices $K_{1}, L_{2}$ and $K_{3}$ solution to the optimization problem (18) subject to the LMI (19) exist, then, $H_{\infty}$ weighting performance is guaranteed for the estimation of the state vector $Y$ and the unknown input $U$.

$$
\min _{K_{1}, L_{2}, K_{3}} \bar{\gamma}
$$

s.t.

$$
\left(\begin{array}{ccc}
\mathcal{N}_{11} & 0 & \mathcal{N}_{13} \\
* & \mathcal{N}_{22} & 0 \\
* & * & \mathcal{N}_{33}
\end{array}\right)<0
$$

with:

$$
\begin{aligned}
& \mathcal{N}_{11}=\left(\begin{array}{ccc}
-K_{1}-K_{1}^{T} & L_{2}^{T} & 0 \\
* & -K_{3} D_{10}-D_{10}^{T} K_{3}^{T}+Q_{1} & I \\
* & * & -\bar{\gamma} Q_{21}
\end{array}\right) \\
& \mathcal{N}_{13}=\left(\begin{array}{ccc}
-\Delta_{1} I & \Delta_{2} I & -\Delta_{3} I \\
\Delta_{1} K_{3} & -\Delta_{2} K_{3} & \Delta_{3} K_{3} \\
0 & 0 & 0
\end{array}\right) \\
& \mathcal{N}_{22}=\left(\begin{array}{ccc}
-\bar{\gamma} Q_{22}+\epsilon_{2} I & 0 & 0 \\
* & -\bar{\gamma} Q_{23}+\epsilon_{3} I & 0 \\
* & * & -\bar{\gamma} Q_{24}+\epsilon_{4}
\end{array}\right) \\
& \mathcal{N}_{33}=\left(\begin{array}{ccc}
-\epsilon_{2} I & 0 & 0 \\
* & -\epsilon_{3} I & 0 \\
* & * & -\epsilon_{4} I
\end{array}\right)
\end{aligned}
$$

The observer's gain $K_{2}$ is computed from: $K_{2}=D_{10}^{T}-$ $L_{2}+K_{3} K_{1}$

The $H_{\infty}$ weighting performance is guaranteed w.r.t. $\dot{U}, \dot{Y}$, $U$ and $v_{x}^{2}$ with the weighting matrices $Q_{1}, Q_{21}, Q_{22}, Q_{23}$ and $Q_{24}$. The attenuation level is $\gamma=\sqrt{\bar{\gamma}}$ which means that:

$$
\tilde{U}^{T} Q_{1} U^{T} \leq \gamma^{2}\left(\dot{U}^{T}, \dot{Y}^{T}, U^{T}, v_{x}^{2}\right) Q_{2}\left(\begin{array}{c}
\dot{U} \\
\dot{Y} \\
U \\
v_{x}^{2}
\end{array}\right)
$$

with $Q_{2}=\operatorname{diag}\left(Q_{21}, Q_{22}, Q_{23}, Q_{24}\right)$.

Remark 1: Note that the diagonal matrices $Q_{21}, Q_{22}, Q_{23}$ and $Q_{24}$ are the weighting matrices that allow us to give more importance to the minimization of a transfer more than another.

Proof: Let us consider the P2WV's longitudinal model (17) and the observer (9). The estimation error dynamic is modeled by the following set of equations:

$$
\begin{cases}M_{10} \dot{\tilde{Y}}= & D_{10} \tilde{U}-K_{1} \tilde{Y}-\delta M_{1} \dot{Y}+\delta D_{1} U-\delta D_{2} v_{x}^{2} \\ \dot{\tilde{U}}= & \dot{U}+\left(K_{3} K_{1}-K_{2}\right) \tilde{Y}-K_{3} D_{10} \tilde{U} \\ & +K_{3} \delta M_{1} \dot{Y}-K_{3} \delta D_{1} U+K_{3} \delta D_{2} v_{x}^{2}\end{cases}
$$

We consider the following Lyapunov function:

$$
V=\tilde{Y}^{T} M_{10} \tilde{Y}+\tilde{U}^{T} \tilde{U}
$$

According to the estimation error dynamics (21), the derivative Lyapunov function is as follows:

$$
\begin{aligned}
\dot{V}= & -\tilde{Y}^{T}\left(K_{1}+K_{1}^{T}\right) \tilde{Y}+2 \tilde{Y}^{T}\left(D_{10}-K_{2}^{T}+K_{1}^{T} K_{3}^{T}\right) \tilde{U} \\
& -\tilde{U}^{T}\left(K_{3} D_{10}+D_{10}^{T} K_{3}^{T}\right) \tilde{U} \\
& +2 \tilde{U}^{T}\left(\dot{U}+K_{3} \delta M_{1} \dot{Y}-K_{3} \delta D_{1} U+K_{3} \delta D_{2} v_{x}^{2}\right) \\
& +2 \tilde{Y}^{T}\left(-\delta M_{1} \dot{Y}+\delta D_{1} U-\delta D_{2} v_{x}^{2}\right)
\end{aligned}
$$

The $H_{\infty}$ weighting performance is guaranteed with the attenuation level $\gamma$ if and only if:

$$
\dot{V}+\tilde{U}^{T} Q_{1} U^{T} \leq \gamma^{2}\left(\dot{U}^{T}, \dot{Y}^{T}, U^{T}, v_{x}^{2}\right) Q_{2}\left(\begin{array}{c}
\dot{U} \\
\dot{Y} \\
U \\
v_{x}^{2}
\end{array}\right)
$$

with: $Q_{2}=\operatorname{diag}\left(Q_{21}, Q_{22}, Q_{23}, Q_{24}\right)$.

The inequality (24) is equivalent to:

$$
\left(\begin{array}{cc}
N_{11} & N_{12} \\
* & N_{22}
\end{array}\right)<0
$$

with:

$$
\begin{aligned}
& N_{11}=\left(\begin{array}{ccc}
-K_{1}-K_{1}^{T} & D_{10}-K_{2}^{T}+K_{1}^{T} K_{3}^{T} & 0 \\
* & -K_{3} D_{10}-D_{10}^{T} K_{3}^{T}+Q_{1} & I \\
* & * & -\gamma^{2} Q_{21}
\end{array}\right) \\
& N_{12}=\left(\begin{array}{ccc}
-\delta M_{1} & \delta D_{1} & -\delta D_{2} \\
K_{3} \delta M_{1} & -K_{3} \delta D_{1} & K_{3} \delta D_{2} \\
0 & 0 & 0
\end{array}\right) \\
& N_{22}=\left(\begin{array}{ccc}
-\gamma^{2} Q_{22} & 0 & 0 \\
* & -\gamma^{2} Q_{23} & 0 \\
* & * & -\gamma^{2} Q_{24}
\end{array}\right)
\end{aligned}
$$

By making the change of variables: $\gamma^{2}=\bar{\gamma}$ and $L_{2}=$ $D_{10}^{T}-K_{2}+K_{3} K_{1}$ and by using the Xie lemma, the last LMI 
is equivalent to the following:

$$
\begin{aligned}
& \left(\begin{array}{cc}
\mathcal{N} 11 & 0 \\
* & \mathcal{N}_{22}
\end{array}\right)+\epsilon_{2}^{-1}\left(\begin{array}{c}
-\Delta_{1} I \\
\Delta_{1} K_{3} \\
0 \\
0 \\
0 \\
0
\end{array}\right) \cdot(*)^{T} \\
& +\epsilon_{3}^{-1}\left(\begin{array}{c}
\Delta_{2} I \\
-\Delta_{2} K_{3} \\
0 \\
0 \\
0 \\
0
\end{array}\right) \cdot(*)^{T}+\epsilon_{4}^{-1}\left(\begin{array}{c}
-\Delta_{3} I \\
\Delta_{3} K_{3} \\
0 \\
0 \\
0 \\
0
\end{array}\right) .(*)^{T}<0
\end{aligned}
$$

Thanks to Schur's lemma, the above inequality is equivalent to (19). Then by minimizing $\bar{\gamma}$, the effect of the transfer from the vector $\left(\dot{U}^{T}, \dot{Y}^{T}, U^{T}, v_{x}^{2}\right)^{T}$ to the unknown input estimation is minimized according to the weighting matrices $Q_{21}, Q_{22}, Q_{23}$ and $Q_{24}$.

\section{Simulation Results}

\section{A. Setup}

The vehicle parameters are as follows: $M_{m}=210 \mathrm{~kg}$, $M_{f}=64 \mathrm{~kg}, C_{d}=0.19 \mathrm{~kg} \cdot \mathrm{m}^{-1}, i_{f y}=0.484 \mathrm{~kg} \cdot \mathrm{m}^{-2}$, $i_{r y}=0.638 \mathrm{~kg} \cdot \mathrm{m}^{-2}, R_{f}=0.274 \mathrm{~m}, R_{r}=0.274 \mathrm{~m}, \alpha=2 / 3$.

The simulations are carried out on a nonlinear model [11] which takes account the combined longitudinal and lateral dynamics of P2WVs. The longitudinal forces are modeled using the magic formula of Pacejka [6] and the data are collected with a sampling rate of $50 \mathrm{~Hz}$. The simulations are carried out for a lane change maneuver where a braking torque is applied to both wheels to reduce the longitudinal velocity of the P2WV from $200 \mathrm{~km} / \mathrm{h}$ to $30 \mathrm{~km} / \mathrm{h}$.

Sensor noises are added to measurements and inputs to get more realistic results. For the scaling of the sensor noise terms, we have used the parameters given in [12]. Zero-mean white noises were added to measurements with the following standard deviations: $\sigma_{a_{x}}=0.23 m . s^{-2}, \sigma_{v_{x}}=0.25 \mathrm{~m} . \mathrm{s}-1$, $\sigma_{\omega_{f}}=0.08 \mathrm{rad} . \mathrm{s}^{-1}$ and $\sigma_{\omega_{r}}=0.08{\mathrm{rad} . \mathrm{s}^{-1}}^{-}$.

For the first observer (the nominal case), $Q_{1}$ and $Q_{2}$ are chosen to be equal to the identity matrix. The obtained attenuation is $\gamma=0.03$.

For the second observer (the uncertain case), the obtained attenuation level is $\gamma=3.16$ and the weighting matrices have been chosen as follows: $Q_{1}=I, Q_{21}=7 \times 10^{-4} I, Q_{22}=$ $6 \times 10^{4} I, Q_{23}=5 \times 10^{-2} I, Q_{24}=0.4$.

\section{B. Results}

In the following, the measured variables are given with curves in blue and the estimated ones with curves in red.

a) Nominal case: In this case, the P2WV's parameters are considered well known. Simulation results are given in figure (2, 3).

The simulation results show that the longitudinal forces and the braking torque are well estimated even with the knowledge of the tire parameters. Note that this scenario involves the nonlinear behavior of tire forces because of the hard braking.

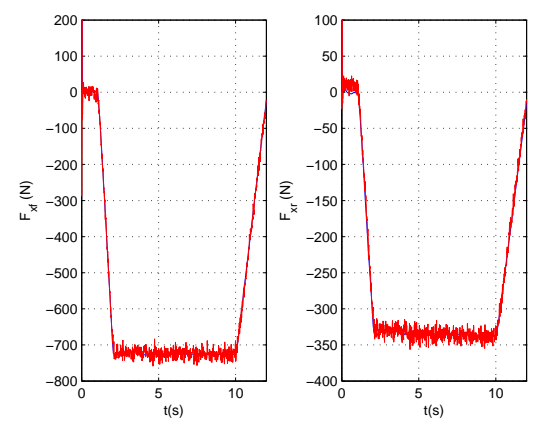

Fig. 2. Longitudinal tire forces

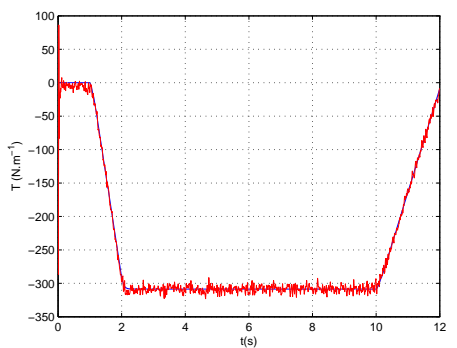

Fig. 3. Equivalent braking torque $T$

Note also the chattering which is observed on the estimation signals. This is due to the derivative action in the adaptation law of the unknown input estimation. By decreasing the gain $K_{3}$ associated with this derivative action, the observer will be less sensitive to noises but with a more delayed response.

b) Uncertain case: In the uncertain case, there is no proof of convergence of the first observer proposed in theorem 1 . So, we will deal with the second observer (theorem 2).

In this case, the mass of the rider is decreased from $64 \mathrm{~kg}$ to $57.6 \mathrm{~kg}(10 \%)$. The radius of the front tire is reduced by $1 \%$ and for the rear tire; the radius is increased by $1 \%$. The aerodynamic coefficient is decreased by $3 \%$. Note that the second observer was designed to minimize the effect of these uncertainties. Figures (4, 5) show that even with uncertainties, the results of simulation remain acceptable.
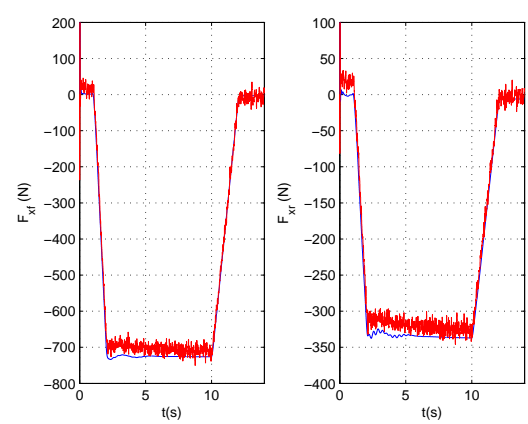

Fig. 4. Longitudinal tire forces with model uncertainties 


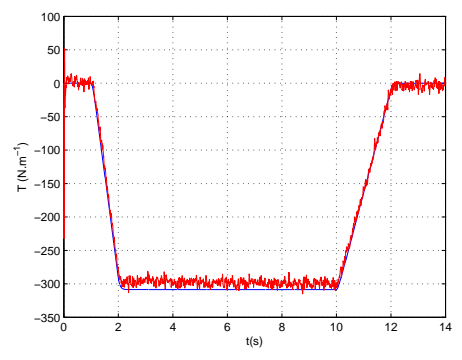

Fig. 5. Equivalent braking torque $T$ with model uncertainties

c) Uncertain case with sudden change in tire-road friction: In this last case, we consider that the parameters are always subject to the same uncertainties. Moreover, we consider that the $\mathrm{P} 2 \mathrm{WV}$ is subject to a sudden change in the tireroad friction at $t=7 \mathrm{~s}$. The results of simulation are given in figures (6, 7).
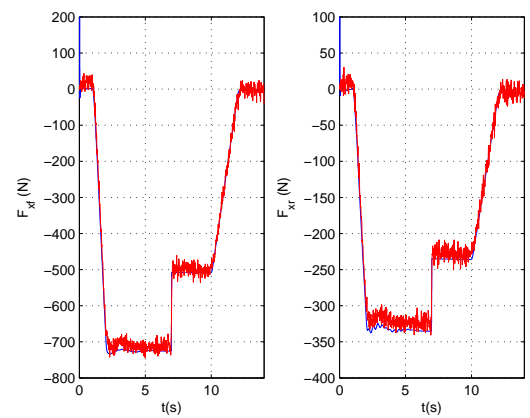

Fig. 6. Longitudinal tire forces with model uncertainties and sudden change in adherence

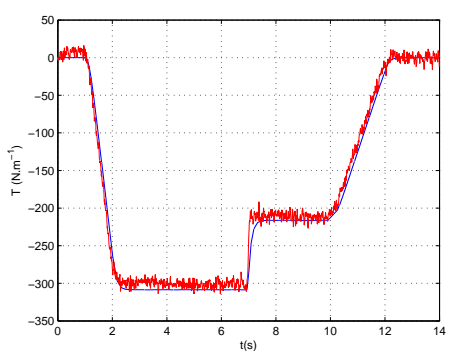

Fig. 7. Equivalent braking torque $T$ with model uncertainties and sudden change in adherence

Even under sudden change in tire-road friction (where the tire parameters and the empirical model will change), the observer still exhibits good performances.

d) Observer evaluation: To test the performances of the proposed observers, we compare the normalized errors in $\%$ which are defined as follows (according to [13]):

$$
\epsilon_{f}=100 \cdot \frac{|u-\hat{u}|}{\max (|u|)}
$$

where $u$ is the unknown input and $\hat{u}$ is its estimate. Table I presents the mean and standard deviation of the normalized error of the unknown input estimation. This confirms the efficiency of the proposed observers and their robustness even with uncertainties and sudden changes in tire-road friction.

TABLE I

PERFORMANCES OF THE OBSERVERS

\begin{tabular}{|c|c|c|c|c|}
\hline \multirow[t]{3}{*}{$\operatorname{Max} \mid F_{x f}$} & $4 N, M c$ & \multicolumn{3}{|c|}{$F_{x r}|=418 N, \operatorname{Max}| T \mid=309 N \cdot m^{-1}$} \\
\hline & Case (a) & Case (b) & Case (b) & Case (c) \\
\hline & $1^{\text {st }}$ obs & $1^{\text {st }}$ obs & $2^{\text {nd }}$ obs & $2^{\text {nd }}$ obs \\
\hline MeanF $F_{x f}(\%)$ & 1.22 & 2.56 & 2.52 & 2.48 \\
\hline MeanF $F_{x r}(\%)$ & 1.35 & 3.25 & 3.25 & 2.93 \\
\hline MeanT $(\%)$ & 1.43 & 2.73 & 2.73 & 3.31 \\
\hline$S t d F_{x f}(\%)$ & 1.56 & 1.96 & 1.72 & 2.05 \\
\hline$S t d F_{x r}(\%)$ & 1.20 & 1.92 & 1.85 & 3.47 \\
\hline $\operatorname{StdT}(\%)$ & 1.90 & 2.99 & 3.23 & 2.25 \\
\hline
\end{tabular}

\section{CONCLUSION AND PERSPECTIVES}

In this paper, an observer was proposed to reconstruct the longitudinal dynamics of two-wheeled vehicles. Without the knowledge of the ratio of braking torque, we proved the undetectability of the longitudinal tire forces. When this ratio is known, the observer allows the reconstruction of the longitudinal tire forces and the engine and/or braking torques without any knowledge of the tire parameters. Simulations through a nonlinear model were given to show the effectiveness of the proposed approach.

\section{REFERENCES}

[1] R. S. Sharp. Stability, control and steering responses of motorcycles. Vehicle System Dynamics, 35:291 - 318, 2001.

[2] A. P. Teerhuis and S. T. H. Jansen. Motorcycle state estimation for lateral dynamics. Vehicle System Dynamics, 50(8):1261-1276, 2012.

[3] L. Nehaoua, D. Ichalal, H. Arioui, J. Davila, S. Mammar, and L. Fridman. An unknown input HOSM approach to estimate lean and steering motorcycle dynamics. Vehicular Technology, IEEE Transactions on, 2013.

[4] D. Ichalal, M. E. H. Dabladji, H. Arioui, and S. Mammar. Observer design for motorcycle lean and steering dynamics estimation: a TakagiSugeno approach. In proc. of the IEEE American Control Conference, 2013.

[5] M. E. H. Dabladji, D. Ichalal, H. Arioui, and S. Mammar. Observer based controller for single track vehicles. In proc. of the IEEE Conference on Decision and Control, 2013.

[6] H.B. Pacejka. Tire and Vehicle Dynamics. SAE International, 2005.

[7] V. Cossalter. Motorcycle Dynamics. Lulu. com, 2006.

[8] M. Corno, S. M. Savaresi, M. Tanelli, and L. Fabbri. On optimal motorcycle braking. Control Engineering Practice, 16:644 - 657, 2008.

[9] J. A. Moreno, E. Rocha-Czatl, and A. V. Wouwer. A dynamical interpretation of strong observability and detectability concepts for nonlinear systems with unknown inputs: application to biochemical processes. Bioprocess and Biosystems Engineering, 37:37 - 49, 2014.

[10] A. Levant. Robust exact differentiation via sliding mode technique. Automatica, 34:379 - 384, 1998.

[11] L Nehaoua, H Arioui, N Seguy, and S Mammar. Dynamic modeling of a two wheeled vehicle : Jourdain formalism. Vehicle System Dynamics, 51(5):648 - 670, 2013.

[12] H. Hamann, J. K. Hedricky, S. Rhodez, and F. Gauterin. Tire force estimation for a passenger vehicle with the unscented kalman filter. In Proc. of the IEEE Intelligent Vehicles Symposium, 2014.

[13] M. Doumiati, A. Victorino, A. Charara, and D. Lechner. A method to estimate the lateral tire force and the sideslip angle of a vehicle: Experimental validation. In Proc.of IEEE American Control Conference, 2010 . 